\title{
Haws-Dieter König Zur Renaissance des autoritären Charakters in den USA
}

"Ich erreiche immer dain die größte Aufmerksamkeit, wenn ich sage, daß wir vielleicht aufbören sollten, uns zu fragen, ob der Rest der Welt uns mag, und daß wir uns dafür entscheiden sollten, wieder in der Welt respektient zu werden, so wie es einmal wark.

Ronald Reagan

\section{Die Irrationalizäi der anserizaniscben Gegensartspolition}

In ihren Studien zum autoritären Charakter hatten Adorno u.a. (1953) in den vierziger Jahren *jenes Potential in der Bevölkerung der Vereinigten Staaten zu ermitteln« versacht, *das in Krisenzeiten« einer dem Faschismus vergleichbaren Bewegung »als aktive Anhänger oder doch als Mitläufer zur Verfügung stehen würde« (Adorno, 1973, S. X f). Daß das empirische Material für diese Untersuchung die Probandengruppe jener $z$ wischen 1910 und 1925 geborenen Mittelschicht bildete, die sin den Großstädten der Westküste« lebt (ebd, , S. XI), ist deshalb bemerkenswert, weil zu Anfang der achtziger Jahre mit Männern wie Reagan, seinem Verteidigungsminister Weinberger und dem Justizminister Smith der zwischen 1911 und 1917 geborene und durch sein Leben in Kalifornien geprägte Amerikaner die Regierungsgeschäfte übernommen hat, der mit seiner die staatlichen Sozialleistungen demontietenden Wirtschaftspolitik das freie Unternehmertum des 19. Jahrhunderts so wiederherzustellen sucht, wie er mit seinem gigantischen Aufrüstungsprogramm zur Politik des kalten Krieges zurückkehrt, um die Sowjetunion »zum Abrüsten zu 'zwingen' ( FR, 11.7 .81$, S. 1).

Die Tatsache, daß in seinem ersten Amtsjahr »zwei von drei Amerikanern Reagans Sparprogramm befürworteten und darüber hinaus »jeder zweite von ihnen ... wie der Präsident weitere Kürzungen« verlangte (Spiegel, 26.10.81, S. 160), verweist datauf, daß dieser Präsident die irrationalen Wünsche jener Mittelschichten angesprochen hat, die ihrer autoritätsgebundenen Persönlichkeissstruktur entsprechend bereit waren, sich auf der Grundlage ihrer Identifikation mit den wirtschaftlich Mächtigen der Aufforderung zu Verzichtleistungen so masochistisch zu unterwerfen, wie sie gleichzeitig den zegen die Herrschenden gerichteten Neid und $\mathrm{Haß}$ sadistisch auf die Armen als die sohnehin schon Getretenenk zu verschieben drängten (Adorno 1973, S. 239). Gewaltige Anstrengungen aller Amerikaner schienen getechtfertigt, weil sich dieses Kleinbürgertum soziale Vorteile davon erhoffte, wenn Amerika nur erst wirtschaftlich und militärisch erstarkt und wieder sunbestritien Nummer eins in der Welt« wäre (Spiegel, 8.9.80, S. 139).

Die Tatsache, daß die Konservativen Reagan aach seinem Wahlsieg zugejubelt hatten, ex habe ajetzt die Chance ... dasselbe zu tun, was Konrad Adenauer einst in Deutschland vollbrachte« (Spiegel, 10.11.80, S. 142), war derart Ausdruck einer gesellschaftichen Kri- 
sensituation, in der die Amerikaner einen Ausweg aus ihrer »Ratlosigkeit und Desorientierung (Mitscherlich und Mitscherlich 1967, S. 34) dadurch suchten, daß sie auf eine »uralte Vaterautorität« zurückgriffen (ebd., S. 22), die nicht wie sein Amtsvorgänger Carter dadurch kompromittiert waren, "gun-shy' - ängstlich im Umgang mit Waffen« zu sein (Spiegel, 28.4.80, S. 129). Denn war Carter unglaubwürdig geworden, der nach Vietnam und Watergate mehr Demokratie zu wagen versuchte und den Menschenrechten in der Politik Geltung verschaffen wollte, dann aber doch aufgrund der sowjetischen Invasion in Afghanistan die Entspannungspolitik preisgab, um schließlich daran zu scheitern, daß e: die Geiseln in Teheran nicht mit Gewalt zu befreien vermochte - so erschien den durch die politische und wirtschaftliche Lage ihres Landes verunsicherten Amerikanern Reagan als eine glaubwürdige Vatergestali, die »Amerika wieder groß zu machen versprach (FR, 29.4.81, S. 3).

Daß Haig drei Monate nach dem Regierungswechsel in Washington feststellte, Amerika sei hente wieder »so selbstsicher wie seit langem nicht mehr《 (FR, 11.5.81, S. 1), weil endlich die »lange Zeit der Gewissensforschung, Unsicherheit und Verwirrung«, »diese Zeit der 'Nabeischau' jetzt zu Ende seik (ebd.), verweist auf das Ausmaß, in dem die von Reagan 1981 betriebene Politik der Stärke Ausdruck einer manischen Selbst-Inszenierung der US $\hat{a}$ war, mit deren Hilfe man auf der Grundlage der Rückkehr zu den soldatischen Tugenden des Heldenmutes und der Opferbereitschaft - die der neue Präsident in seiner Antrittsrede beschworen hatte (vgl. Reagan 1981, S. 10 ff) - die Depression zu überwinden suchie, unter der man aufgrund der seit zwei Jahrzehnten andauernden Krise Amerikas litt. Hatte man sich in dieser Zeit schwach und unterlegen gefühlt, so inszenierte man nunmehr Macht und Stärke, die man dadurch zu beweisen suchte, daß man auch vor einem Krieg nichr mehr zurückschreckt - denn von nun an galt, was das Außenministerium deklamatorisch hatte verlauten lassen: "Wir haben das Vietram-Trauma hinter uns gelassen« (FR, 26.5.81, S. 1).

Wie sehr die von Reagan initiierte Politik der militärischen Konfrontation »Fiktionen« und einem irrationalen »W/unschdenken《 verhaftet ist (Mitscherlich und Mitscherlich, 1967, S. 20), hat in symptomatischer Weise »die massive Militärhilfe« der Vereinigten Staaten für die Junta in El Salvador gezeigt (Spiegel, 2.3.81, S. 131), eine Politik, die Reagan nach seinem Amtsantritt veranlaßt hat und die er auch gegen alle Kritik vtrd Widerstände im eigenen Lande auf der Grundlage der Auffassung verteidigt, daß amerikanischen Waffen und Militärberater jenen Kräften helfen, »die die Menschenrechte unterstützen* (FR, 21.3.81, 3. 2) ${ }^{1}$. Denn indem man heute sein armes Entwikklungslandk wie das von kaum funf Millionen Menschen bewohnte El Salvador (Spiegel, 9. 3.81, S. 139) zum »Schauplatz der Abwehrschlacht gegen den Kornmunismus« macht (ebd., S. 138), obwohl »die gegenwärtige Krise in Mittelamerika in erster linie das Resultat schon lange bestehender sozialer und ökonomischer Ungleichheit und des Fehlens politischer Lösungen ist, nicht aber Folge einer Intervention der Sowjetunion ( $F R, 16.4 .81$, S. 2), setzt Reagan die Politik der Gewalt fort, die in den sechziger Jahten mir dem Krieg in Vietnam zu einem »bedingungslosen Einsatz der gigantischen Militärmaschinerie Amerikas gegen ein unterentwickeltes 22Millionen Volk« geführt hatte (Spiegel, 22.1.73, S. 63), weil man von der »missionarischen Aufgabe überzeugt war, „Südostasien vor dem Kommunismus» bewahren zu müssen (ebd., S. 62).

Angesichts der Irrationalität dieser amerikanischen Gegenwartspolitik stellt sich die Frage, »warum das ganze Land« sich über ein Jahr lang »wie ein Mann erhob, „entschlossen, auch 
unter großen Opfern eine gigantische zusätzliche Aufrüstung ... auf sich zu nehmen (Zeit, 3.4.81, S. 3) - obwohl man annehmen sollte, daß die Amerikaner aus ihren Exfahrungen mit dem Vietnamkrieg und mit Watergate gelernt haben. Daß aber Reagan die Mehrzahl seiner Landsleute für seine Politik zu mobilisieren vermochte, obwohl sie sich gegen ihre eigentlichen Interessen richtet, stellt uns vor ein sozialpsychologisch bedeutsames Problem, das sich vor dem Hintergrund einer Renaissance des autoritären Charakters erklären läßt, der in dem Augenblick wieder an Bedeutung gewinnt, wo es einer politischen Führergestalt wie Reagan gelingt, auf der Grundlage seines weltanschaulichen Angebotes und der von ihm neu inszenierten Mythen der Vergangenheit die neurotischen Wünsche der Massen zu mobilisieren, um zur Macht zu gelangen ${ }^{2}$.

\section{Die Projektion der eigenen Aggression auf die Kommunisten und der Narzißmus der Amerikaner}

Hat Reagan die Russen beschuldigt, daß ssie 'lügen und betrügen' ... und sich eine Moral zurechtgelegt hätten, die es ihnen erlaube, 'jede Art von Verbrechen' zu begehen « (Spiegel, 9.2.81, S. 100), so hat sein Verteidigungsminister Weinberger von der Bedrohung des Weltfriedens durch diesen »aggressiven, imperialistischen, repressiven Staat« gesprochen (Spiegel, 28.9.81, S. 150), der auch nach Auffassug von Haig eine "Politik der Unruhestiftung verfolgt (FAZ, 20.3.81, S. 1), die die Konfrontation mit der UdSSR als »nahezu unvermeidlich« und spermanent« erscheinen lasse (ebd.). Daß aber viele Amerikaner aufgrund der ihnen von Reagan so dramatisch inszenierten Weltlage die Sowjets als Kriminelle ohne jede Moral zu identifizieren anfingen, erlaubte es ihnen, auf diese die Unmoral und die Kriminalität zu projizieren, die sie im Widerspruch zu ihren sozialen Idealen sowohl in ihrem von Egoismus und allseitiger Konkurrenz bestimmten Wirtschaftsleben als auch in der von ihm bestimmten Außenpolitik entwickeln. Denn das abenteuerliche Verhalten, das die USA auf der Grundlage unzähliger Menschenrechtsverletzungen entfalten, indem sie heute Militärdiktaturen wie in El Salvador so selbstverständlich unterstützen, wie sie in der jüngsten Vergangenheit einen Krieg geführt haben, bei dem 55000 Amerikaner und fast zwei Millionen Vietnamesen starben« (Spiegel, 1.2.82, S. 102), spalten sie von der eigenen Wahrnehmung ab und projizieren es auf die Sowjetunion, vor deren $\gg$ Abenteurertum« Reagan deshalb warnt (Spiegel, 25.5.81, S. 124) und die als »der größte Schänder der Menschenrechte in der ganzen Welt« perhorresziert wird (FR, 5.3.81, S. 2).

Gerade weil sie aber infolge dieser Verschiebung ihrer Aggression auf die Kommunisten ihre Feinde dämonisieren, fürchten sie zugleich die Vergeltung der von ihnen denunzierten Feinde.

Im Zuge der sich damit entwickelnden Verfolgungsängste entsteht in Washington die Idee des »inter* nationalen Terrorismus«, der nach Haigs eigenen Worten » 25.5.81, S. 124), eine Unterstellung, die Reagan bekräftigt, wenn er von der Gefahr des aus der Sowjetunion »importierten Terrorismus« warnt (ebd.). Was aber Reagan und Haig in der Gestalt des »internationalen Terrorismus» als eine »Weltgefahr« beschwören, »dessen Bekämpfung eine wichtige Aufgabe für die Außenpolitik» der USA werde (ebd.) - erweist sich als eine in Wirklichkeit unbegtündete Verfolgungsphantasie. Denn eine derartige Gefahr gibt es real nicht, da Terrosismus »auf der Prioritätsliste des FBI erst an 17. Stelle« steht (ebd.), und es nach Auskunft des FBI-Chefs keine »Bewcise fürr sowjetisch geförderten Terrorismus inerhalb der Vereinigten Staaten« gibt (ebd.). 
Die dramatische Inszenierung eines von Moskau aus gesteuerten Terrorismus erweist sich jedoch als eine den herrschenden Interessen dienliche Verfolgungsphantasie, weil sich mit ihrer Hilfe die Massen für eine zu ihren Lasten ausgetragene Innenpolitik mobilisieren lassen, die ihr inneres Gleichgewicht erneut erschüttert, weil ihr Selbstgefühl ohnehin unter der anhaltenden Krise der amerikanischen Wirtschaft - mit einer Inflationsrate von über 14 Prozent und einer Arbeitslosenzahl von über acht Millionen (vgl. Spiegel, 11.8.80, S. 94 und Stern, 19.11.81, S. 278) - gelitten hat.

Wenn aber Reagan davon spricht, daß »die Position der USA heute prekärer als am Tag nach Pearl Harbor« sei (Spiegel, 9.2.81, S. 124), dann funktionalisiert er in dramatischer Weise die Verfolgungsphantasie eines von Moskau aus gesteuerten internationalen Terrorismus im Interesse der wirtschaftlich Mächtigen, um die narzißtische Wut der Massen, mit der sie auf die sich verschärfenden inneren Widersprüche ihres Landes reagieren, auf der Grundlage des Kampfes gegen den Kommunismus neu zu inszenieren, ein Gefecht, das Reagan zur weltpolitischen Seeschlacht zwischen Amerikanern und Russen stilisiert, für die er auch Europa und die Dritte Welt zu mobilisieren sich bemüht.

Die gigantische Aufrüstung gegen die sozialistischen Länder, die Unterstützung rechtsextremistischer Militärdiktaturen im Kampf gegen die nationalen Befreiungsbewegungen der Dritten Welt und schließlich die Gefährdung der Sicherheit Europas aufgrund der von Mechtersheimer so bezeichneten »Europäisierung der Atomkriegsgefahr« (Spiegel, 9.2.81, S. 111) sind Ausdruck eines dramatischen Angriffs Washingtons auf die ganze Mensch heit. Denn die Tatsache, daß Reagan den Beifall seiner Landsleute findet, wenn er angesichts der 200 Millionen Amerikaner die über drei Milliarden Menschen auf der Erde als den »Rest der Welt« bezeichnet (Reagan 1980, S. 29), der wieder lernen müsse, die USA als Großmacht zu srespektieren« (ebd.), verweist auf das Ausmaß, in dem die Amerikaner die westeuropäischen Verbündeten, die Kommunisten und die Menschen der Dritten Welt nicht als Angehörige autonomer Nationen, sondern vielmehr »als Fehler in einer narzißtisch wahrgenommenen Realität« betrachten (Kohut 1975, S. 233).

Denn dadurch, daß die Amerikaner die Sowjets als eine Bande von Mördern und Dieben perhorreszieren, weil sie mit ihrer Gesellschaftsordnung sden Glauben an die liberale Wirtschaft und die liberalen politischen Institutionen als 'natürlichen' ewigen Erscheinungsformen erschüttert« haben (Adorno 1973, S. 271 f), daß sie die nationalen Befreiungsbewegungen der Dritten Welt als »Terrororganisationen« denunzieren (Spiegel, 25.5.81, S. 3), obwohl die Vereinigten Staaten selbst *ein Kind des Aufstandes gegen fremde, ungerechte Herrschaft « sind (FR, 21.3.81, S. 3), und daß sie schließlich Druck auf die Europäer ausüben, indem sie ihnen zum Vorwurf machen, daß sie die US-Politik "nicht aggressiv genug unterstützen (FR, 31.3.81, S. 1) und in einer provinziellen Weise an der Entspannungspolitik festhalten, weil sie nicht »über Europa hinaus« sehen (FR, 4.6.81, S. 1), sondern mit ihrer Friedensbewegung vielmehr »einer sowjetischen Langzeitpropaganda《 unterliegen (FR, 19.10.81, S. 1) - machen sie alle Nichtamerikaner zu einem »widerspenstigen Teil ihres serweiterten Selbst $\alpha$, weil sie davon überzeugt sind, daß sie »das Recht haben, »volle Kontrolle« über sie »auszuüben«, da ihre »bloße Unabhängigkeit«, ja schon ihr »Anderssein ... eine Beleidigung «ür sie darstellt (Kohut 1975, S. 233).

In diesem grandiosen Gefühl der eigenen Überlegenheit gegenüber dem »Rest der Welta, der es versäumt, »den absolutistischen Ansprüchen gemäß zu leben« (ebd.), die Amerika an alle anderen Nationen stellt, werden seine Landsleute auch durch die von Reagan forciert vorangetriebene Aufrüstung bestärkt, für deren neue Waffensysteme sie sich mehr 
und mehr begeistern. Denn gerade weil es den Technologen gelungen ist, die "Sprache des Techrischen gewissermaßen ... in die des Sexuellen zurückzuübersetzen (Anders 1970, S. 107), vermochten sie mit ihren Raketen skollektive Phallik zu produzieren (ebd., S. 101), von denen sich die Massen auch heute wieder wie zur Zeit der Mondlandungen faszinieren lassen. So ist sich Reagan auch des Beifalls seiner Zuhörer sicher, wenn er sich für den erfolgreichen Flug der Raumfähre »Columbia« begeistert und davon spricht, daß »uns« anläßlich dieses Ereignisses »die Welt im Triumphe« sieht (FR, 16.4.81, S. 1). Reagans Worte, der Jungfernflug des Raumgleiters beweise, »daß wir ein freies Volk sind, das große Taten vollbringen kann« (ebd.), erweist sich so als Ausdruck amerikanischer Größenphantasien. Denn auf der Grundlage dieser Begeisterung für die technologischen und militärischen Fortschritte Amerikas wehrt man die Depression manisch ab, die die Bewohner des reichsten Landes der Erde bedroht, weil sie den gesellschaftlichen und politischen Problemen der »zwei Milliarden Menschen, die weit unterhalb jeglicher statistisch fixierbarer Armutsgrenze leben« (ebd., S. 3), so gleichgültig gegenüberstehen, daß sie zu einer Kürzung der Entwicklungshilfe bereit sind, weil "die Völker der Dritten Welt sowieso an Armut gewöhnt seien« (FR, 6.8.81, S. 1).

Auf der Grundlage der Identifikation mit den neuen Waffen, die die Rüstungsindustrie derart im Gewande kollektiver Phalli offeriert, erleben die Amerikaner »eine ungeheure sexualprotzerische Steigerung ihres Selbstbewußtseins« (Anders 1970, S. 104), mit der sich die Allmachtsphantasien ihres grandiosen Selbst durchsetzen, die sich mit den Affekten der narzißtischen Wut amalgamieren, die die Waffen auf sich ziehen, weil sie die Mittel sind, mit denen man sich endlich an den Sowjets für die Kränkungen rächen kann, die die Amerikaner aufgrund der wachsenden sozialen Konflikte im eigenen Lande erfahren.

\section{Der purritanische Glaube als massenbildende Weltanschaunng und die Sauberkeit der amerikanischen Kriegfiibrung}

Als Samuel T. Cohen, der Erfinder der Neutronenwaffe, anläßlich seines Besuches im Vatikan die Gelegenheit hatte, dem Heiligen Vater als „Vater der Neutronenbombex vorge. stellt zu werden (Spiegel, 14.9.81, S. 187), versicherte er Wojtyla auf dessen Frage, ob er denn für den Frieden arbeite, daß er »sein Bestes« getan habe und durch das »Vorbild̋ des Papstes sinspiriert gewesen sei« (ebd.).

Daß Cohen die Arbeit an der von ihm selbst als »scheußliche« Mordwaffe bezeichneten Neutronenbome (ebd., S. 186) in Einklang zu bringen vermag mit der christlichen Maxime der Nächstenliebe, die der Stellvertreter Gottes auf Erden zu predigen weiß, ist nur vor dem Hintergrund der Tatsache verständlich, daß Reagan seine Politik der militärischen Konfrontation gesamtgesellschaftlich durchsetzt, indem er auf der Grundlage einer Rückkehr zu den Tugenden der Pionierzeit den puritanischen Glauben der ersten Siedler wiederbelebt, die in der Neuen Welt *als ein leuchtendes Beispiek der Menschheit zeigen wollten, wie es in einer nur nach dem Wort Gottes lebenden Gemeinschaft zuginges (Kilian 1979, S. 29). Indem Reagan seine Landsleute im Wahlkampf immer wieder an diese Worte von John Winthrop erinnerte (vgl. Spiegel, 19.1.81, S. 95), hat er ihnen signalisiert, daß seiner Auffassung nach die Amerikaner zu einem »Rendezvous mit der Vorsehung erwählt seien (ebd.), dementsprechend wes einen göttlichen Plan gibt, der dieses Land einem Volk von besonderer Bestimmung überschrieben hat« (ebd., S. 94). 
Mit diesem Credo hat Reagan Partei für die »religiöse Front einer neuen politischen Rechten* genommen (Spiegel, 8.9.81, S. 139), die im Wahlkampf an Millionen fundamentalistischer Christen mit dem imperativen Anspruch herantrat, daß Reagan nicht zu wählen »eine Sünde gegen den allmächtigen Gott« sei (ebd.). Denn anders als bei den Christen, die sich heute in der Friedensbewegung politisch engagieren ${ }^{3}$, hat die Religion bei den Neokonservativen in den letzten fünf Jahren deshalb eine restaurative Erneuerung erfahren, weil die Pfarrer angefangen haben, im Radio und im Fernsehen »im Stil von Showmastern« aufzutreten (Spiegel, 22.1.79, S. 134), um für Gott in ihren aus »Unterhaltung, Predigt und Gesundbetung " gemischten Programmen (Spiegel, 8.9.80, S. 139) zu werben. So wie diese religiösen Fanatiker Huxleys »Schöne, neue Welt« und Millers »Tod des Handlungsreisenden« aus dem Unterricht verbannen wollen, so haben sie bereits vereinzelt Bücherverbrennungen organisiert und in 34 von 50 US-Bundesstaaten den Versuch unternommen, »Bücher aus den Bibliotheksregalen zu verbannen« (Spiegel, 29.6.81, S. 121).

Wenn aber Reagan heute sbeinahe so vehement gegen Abtreibung, Empfängnisverhütung und vorehelichen Geschlechtsverkehr zu predigen weiß wie der Papst« (Spiegel, 27.10.80, S. 153), dann kann er der Unterstützung durch die Radio- und TV-Prediger sicher sein, für die »ein Schwächling ist, »wer für Abrüstung eintritt, ein Abartiger, wer Homosexuelle im öffentlichen Dienst toleriert, ein Mörder, wer die Abtreibung befürwortet« (ebd., S. 139).

Diese von Kirche und Staat gemeinsam getragene Offensive gegen Sexualität und Sozialismus verweist auf den Umfang, in dem in der Gegenwart das weltanschauliche Angebot des Puritanismus an die anale Charakterbildung des Kleinbürgertums anknüpft, um als das Ziel von Ordentlichkeit und Sauberkeit die Enneuerung Amerikas so zu bestimmen, wie der anale Sadismus auf die Homosexuellen und die Kommunisten zu verschieben sei.

Um dieser so fanatisch und eigensinnig vorangetriebenen Kampagne gegen den Schmutz und die Unordnung Nachdruck zu verleihen, hat Jerry Falwell, der erfolgreichste aller Fernsehprediger, den Reagan als »moralisches Gewissen der Nation« schätżt, die »Moral Majority« gegtündet, die »anständige Mehrheita, die »ganz im Sinne der regierenden Republikaner für einen 'sauberen Bildschirm', für höhere Verteidigungsausgaben sowie gegen Schwangerschaftsabbruch und Homosexualität" kämpft (Stern, 23.7.81, S. 98).

In seiner sonntäglichen »Old Time Gospel Hour«, die von »273 Fernsehstationen übernommen« wird (Spiegel, 8.9.80, S. 139), greift Falwell zu denselben Worten wie Reagan, indem er gegen die Sowjets als »Monster im Kreml hetzt, die seiner Auffassung nach »vom Himmel nichts zu erwarten« haben, weil sie »wie alle Kommunisten 'Mörder, Betrüger und Lügner' seien (Stern, 23.7.81, S. 98). Dieser Denunziation der Sowjets durch Kirche und Staat entspricht die Idealisierung der amerikanischen Politik, die den Anspruch einlösen soll, den Falwell mit seiner Evangelisation ethebt. Denn Reagan ist der Auffassung, daß die Politik »die höchsten moralischen Erwartungen erfüllen« soll, so daß »die Regierungsgebäude etwas von der moralischen Kraft ausstrahlen ... wie unsere Kathedralen und Kirchen, unsere Tempelk (Spiegel, 27.10.80, S. 164).

Wo aber die sozialen und politischen Zusammenhänge derart vereinfacht werden, daß Amerika als der Verteidiger des Sauberen und Guten idealisiert wird, während man die Gewalt der eigenen Politik aus der Wahrnehmung abspaltet und auf den Feind projiziert, da werden die Russen als übermächtige Verfolger erlebt, so daß man die Angst vor ihnen nur durch die Aufspaltung des bedrohlichen Objekts abzuwehren vermag (vgl. Klein 1962 , S. 105 ff). Denn dadurch, daß man das böse Objekt »in winzige Fragmente zersplit- 
tert und auflöstж (Segal 1964, S. 80), stellen sich die Sowjets für die Amerikaner nur noch als bedeutungslose Objekte dar, die nichts anderes sind als Ungeziefer. Die Kehrseite dieser Abwehr gegen die Verfolgungsängste ist jedoch die Schwächung der Wahrnehmungsfähigkeit des Ichs, weil mit der Fragmentierung des bösen Objekts der Verfolger die Gestalt einer unüberschaubaren Masse »bizarrer Objekte« annimmt (vgl. ebd.), die den Körper und die Seele des Amerikaners so zu vergiften drohen wie sein Land:

Das »Böse« gewinnt »den Anstrich der hygienisch interpretierten Undurchsichtigkeit, in deren Schutz das Unsaubere, Trübe, Anstößige, Ungehörige, Unkorrekte, Recht und Moral Gefährdende schimmelpilz- und ungeziefergleich wuchert, wenn man es nicht rechtzeitig austilgt« (Alsheimer 1966, S. 27).

Gerade weil sich aber dem autoritären Charakter in den USA die Möglichkeit bietet, seine anal-sadistischen Wünsche dem weltanschaulichen Angebot des Puritanismus entsprechend im Dienste eines »Kampfes für das Gute in der Welt« zu funktionalisieren, um »das Böse* auszurotten, ist die Idealisierung einer Politik der militärischen Stärke die Folge. Denn wie in Vietnam wird der Krieg für die Amerikaner zu einer »hygienischen Kampagne« (ebd.),

einer »Sprayaktion mit Insekten- und Unkrautvertilgungsmitteln zu dem $Z_{\text {wecke, }}$ durch die Ausmerzung möglichst vieler 'kommunistischer Schädlinge' im Land wieder 'saubere', 'klare' und 'übersichtliche' Verhältnisse herzustellen« (ebd., S. 28).

Wo aber das Schlachtfeld zur Szenerie einer systematischen »Schädlingsbekämpfung* wird (ebd.), um den »Weg für ein System politischer Hygiene und Rechtschaffenheit« frei zu machen (Marcuse 1968, S. 26), da setzt sich der autoritäre Charakter in seiner gefährlichsten Gestalt durch: Denn dem »manipulativen Typus«, der sich derart Geltung verschafft, fehlen Objektkathexis und emotionelle Beziehungen» in demselben Maße (Adorno 1973, S. 334 f), wie er »alles Technische, alle Dinge, die als 'Werkzeug' benutzt werden können, ... mit Libido besetzt, um »in einer Art $z$ wanghaftem Überrealismus ... alles und jeden als Objekt « zu betrachten, »das gehandhabt, manipuliert und nach den eigenen theoretischen und praktischen Schablonen erfaßt w wird (ebd., S. 335).

Spricht man die dem manipulativen Typus eigene »nüchterne Intelligenz und die fast komplette Absenz von Affekten* im allgemeinen Faschisten wie Himmler zu (ebd.), so ist es im Zusammenhang unserer Überlegungen zumindest bemerkenswert, daß auch der neue amerikanische Präsident mit »totalitären Lösungen« sympathisiert, wie es sein Auftreten zu Anfang des Vietnamkrieges dokumentierte, als er ein probates Mittel zur Beendigung des Krieges gefunden zu haben glaubte:

„Wir sollten Nordvietnam den Krieg erklären. Dann könnten wir bis Mittag das ganze Land einebnen und wären zum Abendessen wieder zu Hause« (Reagan 1980, S. 32).

Wenn auch der Wunsch, mit Vietnam an einem einzigen Tag fertig zu werden, Ausdruck einer Größenphantasie war, die an det Realität des vietnamesischen Widerstandes scheitern sollte, so wurde doch die Größenidee, das Land einzuebnen, zumindest in erheblichem Umfang Wirklichkeit, als man Vietnam durch 28 Millionen Bomben und Granaten verwüstet (vgl. Spiegel, 22.1.73, S. 64) und seine wertvollen Edelholzwälder mit großen Bulldozern niedergewalzt hatte (vgl. Spiegel, 29.1.73, S. 69).

Der vom analen Sadismus bestimmte Beseitigungswunsch (vgl. Abraham 1924, S. 122), 
Vietnam einzuebnen und es zum Fußboden ${ }^{4}$ einer mit Hilfe modernster Technologien systematisierten Aktion des »Aufräumens« zu machen (vgl. ebd., S. 123), um dieses Land mit seinen undurchsichtigen und trüben Dschungeln »vom Feinde« zu »säubern« (ebd.), ist Ausdruck einer den manipulativen Typus faszinierenden Kriegführung, die mit der Entwicklung der Neutronenwaffe einen neuen Höhepunkt erreicht hat. Zwar hatte es schon die moderne Rüstungstechnologie ermöglicht, das Schlachtfeld zum Szenarium einer hygienisch begründeten »Massensäuberung des guten Gewissens« zu machen (Marcuse 1968, S. 26), weil mit dem Militärflugzeug und der Rakete »die menschlichen Opfer ... der Wahrnehmung des Täters« so entrückt sind (ebd., S. 25), daß sie tatsächlich nur noch als unter Beschuß genommenes Ungeziefer erscheinen. Jedoch läßt sich die »Ideologie der Schädlingsbekämpfung« (Alsheimer 1966, S. 28) mit der Neutronenbombe in einer neuen Weise in die Wirklichkeit übersetzen, weil es sich nach Auffassung der Amerikaner um eine »saubere Kernwaffe handelt, die anders als die traditionelle Nuklearwaffe zwar »die Menschen tötet, aber Eigentum verschont« (Spiegel, 14.9.81, S. 186).

Gerade weil »statt zerstörter Städte dahinsiechende Menschen und kranke Natur die Folgen ihres Einsatzes sind (Spiegel, 17.8.81, S. 28), entspricht die Neutronenwaffe den Bedürfnissen des manipulativen Typus: »sauber erscheint ihm diese Waffe deshalb, weil sie bloß die ihm gleichgültigen Menschen tötet, während sie den materiellen Besitz verschont, der ihm seiner analen Charakterbildung entsprechend allein als wertvoll erscheint. Zwar sind »die biologischen Zerstörungen bei der Neutronenwaffe um so verheerender« (ebd.), weil im weiteren Umkreis des unmittelbaren Zentrums der Neutronenexplosion der Tod erst nach Stunden oder Tagen, in den schlimmsten Fällen gar erst »nach einigen Wochen langsamen, äußerst schmerzhaften körperlichen Verfalls« eintritt (ebd.). Jedoch tritt die Bedeutung dieser Tatsache hinter den alle Aufmerksamkeit auf sich ziehenden Sachverhalt zurück, daß die Kriegsführung nunmehr zu einer Funktion eines phänomenalen Zusammenwirkens technologischer und mikrobiologischer Errungenschaften wird: Was bisher eine hygienische und moralische Kampagne war, die zur Ausrottung des feindlichen Ungeziefers erforderlich war, gewinnt nunmehr die Autorität eines medizinischen Eingriffs. Denn die Menschen werden nicht mehr wie bei der konventionellen Atombombe durch die Druckwelle zerfetzt und durch die Hitze verbrannt, sondern werden wie in einem Labor $»$ versaftet $\ll$ (»juiced $\ll$ ) (ebd.) - weil die Folgen der Neutronenexplosion vergleichbar sind »mit Milliarden kleiner Injektionen hochwirksamer Säure« (ebd.).

Gerade weil der Terror und die Greuel, den die Amerikaner mit den von ihnen entwickelten Waffen im wachsenden Umfang anzurichten imstande sind, mit ihren demokratischen und sozialen Idealen so unvereinbar ist, läßs sich diese Diskrepanz nur dadurch überbrücken, daß man den Anschein der Sauberkeit und Hygiene dieser Waffen auch dadurch erzeugt, daß man den Kriegsschauplatz in ein medizinisches Laboratorium verwandelt. Wo nämlich davon gesprochen wird, daß die Neutronenwaffe »eigentlich ein riesiger Röntgenapparate sei (Spiegel, 14.9.81, S. 186), und wo man die neuen Mittelstreckenraketen als Mittel betrachtet, um im Krisenfall von Westeuropa aus »chirurgische Einzelschläge gegen ausgewählte militärische und politische Ziele« wie den Kreml anzudrohen (Spiegel, 9.2.81, S. 110), den man damit »aus dem Antlitz der Erde herausoperieren« könne, so daß sich »der 'Nebenschaden' selbst in Moskau in Grenzen hält« (ebd., S. 111) - da setzt sich der falsche Schein durch, als ob die militärischen Operationen bloß prophylaktische und therapeutische Maßnahmen darstellen, die der Abschreckung dienen und auch den Feind vor größerem Schaden bewahren sollen. 


\section{Massenbildung auf der Grundlage der um den Cowboy entstandenen Mytbenbildung}

Ausgerechnet am 36. Jahrestag des nuklearen Angriffs auf Hiroshima, als die Japaner in einer Feierstunde im Friedenspark dieser Stadt jener Katastrophe gedachten, um nicht zuletzt »die Namen von weiteren 2753 Menschen, die in den vergangenen zwölf Monaten den Folgen der Strahlenschäden erlagen beziehungsweise erst jetzt als Opfer des amerikanischen Angriffs bekannt geworden waren, auf der Gedenktafel des schwarzen Ehrenmals« einzutragen (FR, 7.8.81, S. 1) - gab Ronald Reagan das Signal zum Bau der Neutronenbombe (vgl. FR, 10.8.81, S. 1 und FR, 14.8.81, S. 1). Signalisierte Reagan mit dieser Geste der ganzen Welt, daß die Amerikaner schon 1945 bewiesen haben, daß sie keine Skrupel kennen, Nuklearwaffen einzusetzen, wenn es darum geht, einen Feind zu vernichten, der ihnen wie heute schon wieder die Sowjets ein Pearl Harbor aufzwinge, so vermochte er die Amerikaner mit diesem Verhalten gerade auch deshalb für sich einzunehmen, weil er diese politische Entscheidung vor Journalisten rechtfertigte, indem er sich am Gartentisch vor seiner Ranch als Westerner in Szene setzte, der seinen Erläuterungen mit einem breiten Lächeln und mit weit ausholenden Gesten seiner Hände und Arme über die vor ihm stapelweise angeordneten Papiere Nachdruck verlieh (vgl. Spiegel, 17.8.81, S. 17).

$\mathrm{Daß}$ unter Reagans Choreographie die politische Entscheidung für die Neutronenbombe zu dem Entschluß eines Cowboys wurde, der an der Grenze zur entlegenen Wildnis Kaliforniens noch als Präsident Holz hackt, ein Stück Weg rodet und auf seinem Pferd ausreitet (vgl. Spiegel, 19.1.81, S. 93), mußte auch deshalb als ein Beweis für »Leadership« gegenüber den Widerständen im eigenen Lande und der Kritik in Europa imponieren (Spiegel, 17.8.81, S. 22), weil er auf diese Weise den Wünschen und Träumen all der $\gg$ Büroangestellten und Geschäftsleute entsprach, die heute »wie einst die Law-and-Order-Helden aus Wildwest ... von Los Angeles bis New York Stiefel und Hut火 tragen (Stern, 30.4.81, S. 28).

Gerade weil die Amerikaner heute den Westerntrend zur Mode machen und dem CowboyFilm eine Renaissance ermöglichen (vgl. Stein 1981, S. 25), um ihr durch die gegenwärtige Krise Amerikas gestörtes Selbstgefühl zu restituieren, fällt es ihrien leicht, ihr Land als das »Marlboro Country« zu identifizieren (vgl. z.B. Newsweek, 13.4.81, S. 31 oder Newsweek, 20.4.81, Rückseite). Denn hierbei handelt es sich um den Namen für eine Inszenierung, die suggeriert, daß der Amerikaner sein Glück in der entpolitisierten, aber für einen Zigarettenkonzern profitablen Gestalt einer Privatsphäre findet, in der er die »Freiheit unbeschränkten Handelns« (Lorenzer 1981 a, S. 170) als ein Cowboy entdecken kann, weil ihm eine »Regression ins Passiv-Genußvolle« die Marlboro ermöglicht, mit der sich die Sorgen um die soziale und politische Realität so verflüchtigen wie der Rauch dieser Zigarette. Gerade weil diese Bilderwelt den Marlboro-Helden in der wirreal fernen Landschaft" einer noch unbetretenen Natur ansiedelt, wirkt sie als »abstraktes Gegenbild zu den täglich erfahrenen Freiheitseinschränkungen« (ebd.), indem sie Autonornie veêheißt, sobald sich der Alltagsmensch mit Hilfe der Zigarette als Westerner in Szene setzt.

Mit der Amtsübernahme des neuen Präsidenten ist diese Szener ie politisiert worden: Denn wie es in der Wahlkampfzeit auf den Plakaten versprochen wuxde, die Reagan mit Cowboyhut abbildeten, wird dieses Amerika von Tag zu Tag mehr zum »Reagan Country« (Stern, 30.4.81, S. 26 oder auch Ferguson und Rogers 1981, S. 78). Die Verheißung eines »Reagan Country bedeutet aber, daß es dem neuen Präsidenten gelungen ist, eine sillusionär-symbiotische Szenerie« zu schaffen (Lorenzer 1981 a, S. 170), die er selbst in der bildhaften 
Gestalt des Westerners beherrscht, eine neue Vaterfigur, die die Massen zu ihrem kollektiven Ich-Ideal erhoben haben, weil er sich als der von ihnen bewunderte Cowboy in Szene setzt, den er in zahlreichen Western-Filmen geschauspielert hat. Das bedeutet aber, daß Reagan sein wirtschaftliches und militärisches Programm durchsetzt, indem er die Masse nicht nur über das Über-Ich durch die Erzeugung von Angst und Schuldgefühlen auf der Grundlage einer Wiederbelebung der zur Weltanschauung verkommenen puritanischen Religion anspricht, sondern sie auch über das Ich-Ideal durch den Rückgriff auf illusionäre Hoffnungen und falsche Idealbildungen auf der Basis der »Verführungsgewalt der Bilder» für sich einnimmt (Lorenzer 1981 b, S. 17) $)^{5}$, indem er sich als der in der Bilderwelt des Westens idealisierte Frontier der Pionierzeit in Szene setzt.

Folgt Reagan mit dieser Selbst-Inszenierung als Cowboy dem Beispiel Roosevelts, der in den dreißiger Jahren das infolge der Weltwirtschaftskrise depressiv verarmte Selbstgefühl seiner Landsleute auf der Grundlage einer Rückkehr zu den Tugenden des Westerners zu erneuern vermochte (vgl. Seeßlen, Weil 1979, S. 80 f), so erklärt sich doch die Tatsache, daß heute die auf die ersten Siedler zurückgehende puritanische Weltanschauung und die um sie entstandene Bilderwelt ${ }^{6}$ schon wieder Inszenierungen ermöglichen, die Reagans Politik zu beschönigen und rechtfertigen imstande sind, dadurch, daß sie schon in den Gründerjahren im Dienste einer von Gewalt bestimmten Politik funktionierten. Denn die mit der Landnahme und Urbarmachung des nordamerikanischen Kontinents entstandenen „Pseudomythen« um den Cowboy (Lorenzer 1981 a, S. 119) und die von den Neuengländern entwickelte Gestalt der puritanischen Religion sind nicht einfach Ausdruck einer Geschichte Amerikas, die vom »Ethos der Demokratie und der Gleichheit« getragen wäre (Jacobs, Landau, Pell 1971, S. 32); vielmehr sind sie Ausdruck einer Idealisierung, mit deren Hilfe man die Tatsache abwehrt, daß diese Geschichte der Gründerjahre zugleich durch eine »Politik des Rassenhasses und Völkermordes« bestimmt wurde (ebd.). Denn für die Entfaltung Amerikas zur Großmacht war die Versklavung der aus Westafrika deportierten Schwarzen so konstitutiv wie der Massenmord an den indianischen Völkern ${ }^{7}$.

Der amerikanische Holocaust, dem die Ureinwohner der Neuen Welt zum Opfer fielen, war nämlich das Resultat einer Politik des Schreckens, für deren unmittelbare Gewalt »der Dauereinsatz der eigens für den Indianerkampf geschaffenen U.S.Cavalry« (Spiegel, 19.11.79, S. 216) so typisch war wie das Kopfgeld für einen gefangenen oder getöteten Indianer, das 100 Dollar betrug - gleichgültig, ob es sich um Krieger, Frauen oder Kinder handelte (vgl. Biegert 1976, S. 21).

Hatte der freundliche Indianer die ersten Siedler »vor dem Hungertodk gerettet (Jacobs, Landau, Pell 1971, S. 41), indem er sie lehrte, wie man Mais und andere grundlegende Nahrungsmittel anbaut (vgl. ebd., S. $44 \mathrm{f}$ ), so schien er ein sheidnisches Paradies« zu verheißen, »von dessen Wiedergewinn die Amerikaner in den Wäldern der Neuen Welt geträumt haben, ein naturhaftes Eden, das mit dem einbrechenden Christentum verloren ging (Fiedler 1968, S. 135). War derart für den in die Wildnis verpflanzten »W ASP (White Anglo-Saxon Protestant)« (ebd., S. 13) der Indianer »ein radikal fremdes Wesen« (ebd., S. 25), der ihn in schmerzlicher Weise daran erinnerte, daß das von ihm geschaffene Selbst, »das immerzu sich bezwingt«, darüber »das Leben versäumt« (Horkheimer, Adorno 1944, S. 52), so wurde der Wilde für den weißen Amerikaner zum Symbol dafür, was dieser aufgrund seiner auf „Gehorsam und Arbeit» beruhenden Zivilisation (ebd., S. 33) als sündhafte Sinnlichkeit tabuierte. Mit der Eroberung Amerikas hatte dieser WASP damit die Möglichkeit gefunden, den inneren Abwebrkampf seines Ichs gegen die tabuierten 
Triebansprüche im Kampf gegen den Indianer an der Grenze zur Wildnis in Szene zu setzen.

Die allgemein verbreitete Phantasie, daß der Indianer nur darauf aus sei, die weißen Mütter zu vergewaltigen und ihre Säuglinge zu erschlagen (vgl. ebd., S. 49 und S. $106 \mathrm{ff}$ ), bringt den auf den Indianer projizierten Inzestwunsch mit der Mutter in demselben Maße zum Ausdruck, wie den auf ihn projizierten Wunsch »nach der Schändung der weißen weiblichen Unschuld (ebd., S. 108), mit dem sich die Amerikaner an den Frauen dafür zu rächen suchen, daß sie ihnen die Lust verweigern, die sie als Mutterfiguren nicht gewähren dürfen.

Gerade weil aber der in Wirklichkeit »sexuell zurückhaltende Indianer (ebd., S. 49) aufgrund der auf ihn projizierten Wünsche des WASP als zügellos seine Sexualität auslebender Wilder identifiziert wurde, verletzte er das Gesetz des Vaters, das der Weiße an der Grenze zur Wildnis zu verteidigen suchte. Der Schmerz des Frontiers, um die Sexualität betrogen worden zu sein, wurde so zur Lust, als er sich als strafender Vollstrecker der väterlichen Ordnung in Szene setzte: Indem er die aufgrund seiner Sexualängste stets befürchtete Kastration durch die Kastration der Ureinwohner inszenierte, eine Kastration, die nur mit Mühe dadurch verhüllt wurde, daß sie sich vom Genitale auf das zu skalpierende Haupthaar des Indianers verschob. Doch schon bald projizierten die Siedler der englischen Kolonien auch diese von ihnen verübte Grausamkeit auf den »bösen Indianer« und "hielten das Skalpieren ... für ein völlig unbegreifliches Verbrechen der Ureinwohner, obwohl es in Wirklichkeit bei den Indianern gar nicht Brauch war, bis der weiße Mann für Erschlagene Prämien auszusetzen begann« (ebd., S. 46).

Auf diese Weise gewann der Amerikaner seine neue Identität dadurch, daß er als Europäer aufgrund seiner Auswanderung die Möglichkeit gewann, in der Weite des Westens noch einmal die Gewalt in Szene zu setzen, mit der das ihm von der Zivilisation auferlegte Über-Ich sein Ich in der Enge des psychischen Raumes gezwungen hatte, die Triebansprüche zu disziplinieren und eine anale Charakterbildung zu ermöglichen, um dem weltanschaulichen Angebot des protestantisch-kapitalistischen Geistes entsprechend Arbeitsamkeit und Ordentlichkeit, Besitzgier und Sauberkeit zu entwickeln (vgl. Fromm 1970, S. 59* 68). Aus diesem Grunde wurde für die Weißen der Völkermord an den Indianern zu einem Mittel, um sich dafür zu rächen, was ihnen die westliche Zivilisation angetan hatte. Die Verleugnung und Verdrängung der Geschichte dieses amerikanischen Holocaustes gelang aber in dem Maße, wie man im Rückgriff auf »die maximal 35000 realen Cowboys der Jahrhundertwende« (ZEITmagazin, 10.4.81, S. 10) eine Bilderwelt erfand, die mit dieser Vergangenheit zu versöhnen imstande war: Diese Viehtreiber, die nichts anderes waren als

»ausgemergelte, arme Schlucker aus Irland und Galizien, Einwanderer aus Mexico und Armenien, Flüchtlinge aus den ausgepowerten Südstaaten (unter ihnen rund 8000 Schwarze) und dem Nirgendwo von New York City« (ebd.),

wurden zum Gegenstand einer Mythenbildung, mit deren Hilfe die Siedler zur Zeit der Eroberung und Besiedlung des Westens nachträglich zu idealisierten Cowboys gemacht wurden, denen ein »sorgfältig austariertes Cowboy-Image« verliehen wurde, das als erster William F. Cody alias 'Buffalo Bill' in alle Welt verkaufte (vgl. ebd.).

$\mathrm{Daß}$ aber diese „Gewalt« des Frontiers, die »einstmals in den leeren Weiten« des nordamerikanischen Kontinents »tolerierbar« gewesen sei, sich in der Gegenwart $\gg$ zurückgewandt habe gegen das moderne Amerika, wo es mit dem Drauflos-Schießen weitergeht« (Time, 
13.4.81, S. 34), ist eine ins öffentliche Bewußtsein so selbstverständlich eingegangene Auffassung, daß sie auch noch ein Journalist anläßlich des Attentats auf Reagan vertreten hat, um für die Forderung nach Waffengesetzen Partei zu ergreifen. Dieses Beispiel verdeutlicht aber den Einfluß der um den Cowboy entstandenen Mythenbildung auf das Alltagsbewußtsein, eine Bilderwelt, die den Massenmord an den Indianern entwirklicht, indem sie die Neue Welt zu einem Land der »leeren Weiten«stilisiert, das erst die Siedler bevölkert zu haben scheinen. War die von den Amerikanern idealisierte Gewalt der Pioniere in Wirklichkeit durch den an den Indianern begangenen Holocaust ihres verbrecherischen Charakters überführt worden, so suchte der Pseudomythos um den Westerner auf der Grundlage dieser die menschliche Existenz der Ureinwohner ausradierenden Inszenierung nach einer seinen Interessen angemessenen Erklärung dafür, weshalb die Gewalt des Westerners heutzutage unglaubwürdig geworden sei: Sei die Gewalt der Cowboys auf dié »menschenleeren Weiten« der Prairien zugeschnitten gewesen, wo die Waffe im Kampf gegen die Wildnis unerläßlich gewesen sei, so haben sie in der Enge der Städte angefangen, sich »gegenseitig zu bedrohen und beim geringsten Anlaß zu zerfleischen« (Seeßlen, Weil 1979, S. 140). Zu betrauern sei deshalb im Rückblick auf die Gründerzeit nicht der Tod der Indianer, die dem von den Siedlern organisierten Genozid zum Opfer fielen; vielmehr sei das Schicksal des Westerners zu beklagen, der mit der Entstehung der Städte zum Sterben verurteilt gewesen sei.

Der Unfähigkeit zur Trauer um den Tod der von den Weißen ausgerotteten Indianer entspricht so die melancholische Klage um den Tod des Cowboys, der schon immer ein einsamer Held gewesen und nunmehr, eingeholt durch den gesellschaftlichen Fortschritt, zur tragischen Gestalt geworden sei. Daß die Cowboys sich in den Städten am Ende selbst zerfleischen, bedeutet deshalb nichts anderes als die in der Bilderwelt des Westerners in Szene gesetzte »Selbstzerfleischung der Melancholie« (Mitscherlich und Mitscherlich 1967, S. 79), aufgrund derer die Amerikaner in der Konfrontation mit dem Indianer nur den seigenen Verlust an Selbstwert« beklagen (ebd., S. 78), obgleich nur die Trauer um die Opfer ihrer Gewalt eine Bewältigung der Vergangenheit ermöglicht hätte, ein Verlust, der auch deshalb bedeutsam ist, weil mit dem Mord an den Indianern etwas getötet wurde, »was für die Neue Welt von vitaler Bedeutung« war, eine Vernichtung dessen, »was Amerika ... bis zu diesem Moment gewesen« war (Fiedler 1968, S. 87).

So wie die Bilderwelt um den Cowboy, der durch die leeren Weiten der Prairien reitet, den Massenmord an den Indianern entwirklicht, so vermag Reagan seine Landsleute auch dadurch für den Bau der Neutronenbombe einzunehmen, daß er diese politische Entscheidung auf seiner Ranch als der Westerner einer entlegenen Wildnis trifft, die die Millionen von Menschen vergessen läßt, die durch den - militärisch allein wirksamen - Einsatz von Hunderten von Neutronenbomben zu einem langwierigen und schmerzhaften Tod verurteilt wären (vgl, Spiegel, 17.8.81, S. 29). Stattdessen drängt sich den Amerikanern mit Reagans Selbst-Inszenierung als Cowboy das Bild eines heldenhaften Mannes auf, unter dessen Regie es nur darauf ankommt, ein starker Präsident zu sein, der im Ernstfall das Ieben einer Unzahl seiner (europäischen) Schützlinge so bereitwillig opfern würde, wie der Westerner immer schon »so etwas wie eine Mutter ... und eine Amme derselben Tiere ist, die er ihrem frühen Tode zuführt« (Erikson 1950, S. 297). Denn gerade weil das ungelöste Problem des amerikanischen Mannes in seiner infantilen Abhängigkeit von der einstmals als gefühlskalt und herrschsüchtig erlebten »Mom\& liegt (vgl. ebd., S. 282 ff) - jener Mutter, die in ihrem Verhalten gegenüber ihrem Kind die Gewalt reproduziert, die in der Gesell- 
schaft die wirtschaftlich Mächtigen heute im Arbeitsprozeß mit derselben Rücksichtslosigkeit ausüben, mit der sich die Frontiers einst die Natur der Neuen Welt unterwarfen ${ }^{8}$-, sucht er sich zwanghaft der Überlegenheit und Männlichkeit des Cowboys dadurch zu vergewissern, daß er wie Reagan auf der Grundlage der Rationalisierung, es gäbe »Dinge«, »für die Männer zu sterben bereit sein müssen« (Spiegel, 19.1.81, S. 96), so selbstverständlich über das Leben seiner Mitmenschen verfügt, wie er sich früher seiner Mutter hilflos ausgeliefert fühlte.

Indem aber Reagan auf der Grundlage seiner Selbst-Inszenierung als Cowboy davon spricht, daß John Wayne sder ganzen Welt eine Vorstellung von dem« gegeben habe, »was ein Amerikaner sein sollte« (Reagan 1979, S. 116), idealisiert er wie dieser die Totschläger und Mörder, denen die Indianer zum Opfer gefallen sind. Denn der bekannte WesternDarsteller ist der Überzeugung, daß wir nicht »im Unrecht waren, als wit« den Indianern »dieses große Land weggenommen haben«, weil sie »so eigennützig« waren, »es für sich behalten zu wollen« (Biegert 1976, S. 15). So wie aber für Männer wie Wayne oder Reagan die Henker der Pionierzeit nichts anderes als Helden waren, so bewundern sie auch »die das Wort Killer als Ehrentitel betrachtenden »Green Berets» (Spiegel, 17.7.78, S. 68), jene US-Elitetruppen, über deren Kampf in Vietnam John Wayne einen Film gedreht hat, für den sich noch vor zweieinhalb Jahren Reagan begeistert hat (vgl. Reagan 1979, S. 118). Die Politik der Stärke, die der amerikanische Präsident im Rückgriff auf die um den Cowboy entstandene Bilderwelt gesamtgesellschaftlich über ein Jahr lang durchgesetzt hat, erhebt damit zum kollektiven Ich-Ideal seiner Landsleute den Amerikaner, der als Frontier die Indianer so skrupellos tötete, wie er als Soldat der »Green Berets« in Vietnam gekämpft hat und heute die Nationalgardisten El Salvadors im Kampf gegen die Befreiungsfront dieses Landes ausbildet (vgl. Spiegel, 1.2.82, S. 102).

\section{Die Angst vor dem Weltuntergang in Amerika}

James Watt, der neue US-Innenminister, dem die Aufgabe zufällt, die in den letzten zehn Jahren vom Repräsentantenhaus verabschiedeten »Gesetze zur Erhaltung der Umwelt« wieder rückgängig zu machen, hat im Kongreß auf die Frage »nach seiner grundsätzlichen Bewertung von Umweltschutz und Landschaftserhaltung« (FR, 6.7.81, S. 3) folgende Antwort gegeben: »Ich weiß nicht, auf wie viele Generationen wir noch rechnen können bis zur Wiederkunft des Herrn« (ebd.). Vermag Watt der Sorge der Umweltschützer »vor einer Zerstörung des Landes« (ebd.) dadurch entgegenzutreten, daß er die begründete Furcht vor einer ökologischen Katastrophe auf der Grundlage seines fundamentalistischen Glaubens abwehrt, sie verleugnet und sie stattdessen als die Sorge eines Christen um das Gottesreich in Szene setzt, das demnächst auf der Erde zu erwarten sei, so versuchen viele Amerikaner auch die Angst vor der atomaren Katastrophe, die aufgrund der von Reagan betriebenen Konfrontationspolitik verstärkt wird, dadurch zu entwirklichen, daß man sie ebenso als gerechtfertigte Furcht vor dem Jüngsten Gericht dramatisiert.

So wie aber Schreber auf dem Höhepunkt seiner Krankheit eine Phantasie vom »Weltuntergang《 entwickelte (Freud 1911, S. 191), die nichts anderes war als »die Projektion« einer »innerlichen Katastrophe« (ebd., S. 192 f), so entwickelten eine Vielzahl der Amerikaner auf dem Höhepunkt der gesellschaftlichen und politischen Krise ihres Landes die Phantasie einer bevorstehenden Endzeitkatastrophe, die Ausdruck der auf den Himmel der Gläubi- 
gen projizierten Angst vor der nuklearen und ökologischen Katastrophe ist. Das Ausmaß dieser Angst vor dem Weltuntergang zeigt sich darin, daß eine neue Zeitschrift mit dem Titel »Endzeit-Nachrichten« entstand, und man einen »wöchentlichen 'prophetischen Nachrichtendienst' per Telefon « einrichtete. Übertroffen wurde diese Tatsache nur noch durch die »Flut von Filmen über im Neuen Testament vorausgesagte Menschheitskatastrophen« (FR, 18.5.81, S. 1).

Unter diesen Umständen hat Falwell sicherlich allen Grund dazu, sich darauf zu berufen, daß der neuen amerikanische Präsident »wie er selbst ... an die 'baldige Wiederkehr des Herrn'\& glaube (Stern, 23.7.81, S. 100): »'Ich denke häufig', so zitiert er Reagan aus einem Gespräch unter vier Augen, 'daß wir uns rasend schnell auf das Jüngste Gericht zu bewegen'" (ebd.).

Auf der Grundlage der Fernseh-Inszenierungen der Kirche und der Aufrüstungs-Inszenierungen des Staates haben derart die weltliche und die geistliche Macht Rituale organisiert, die sich »nicht zufällig ... immer wieder zu einem Todesszenarium«zusammenschließen (Lorenzer 1981a, S. 168f.). Denn aufgrund von Washingtons Mobilmachung der Amerikaner gegen die ganze Welt wird der Einzelne sunter Vernichtung seiner Individualität als bloßer Teil einer Masse« definiert (ebd., S. 169), ein Verlust, für den die stodessüchtigen Verschmelzungsphantasie« zu entschädigen verspricht (ebd., S. 125), die als die von diesen Menschen erwartete Endzeitkatastrophe Gestalt angenommen hat.

Gerade weil es in der Gegenwart so offensichtlich ist wie niemals zuvor, daß nur gesellschaftliche und politische Veränderungen das unaufhaltsame Fortschreiten in die Katastrophe aufhalten und stoppen können, hatte die Mehrzahl der Amerikaner mit Reagan sympathisiert, um auf der Grundlage der Rückkehr zu den Werten einer längst überholten Vergangenheit die herrschende Ordnung zu verteidigen. Denn daß »alles geändert werden müsse« (Adorno 1973, S. 234), ist eine dem autoritären Charakter unerträgliche Vorstellung, weil sie seine "fundamentale Achtung vor dem Bestehenden« verletzt (ebd.): Eher soll die Welt untergehen, als daß die Ordnung der liberalen Wirtschaft erschüttert wird.

Die Erfüllung der irrationalen Endzeit-Visionen des Jahres 1981 schienen aber in Aussicht zu sein, als der Jungfernflug des US-Raumfähre »Columbiak gelungen war. Denn der erfolgreiche Probeflug des »wiederverwendbaren Raumtransporters« (Spiegel, 30.3.81, S. 156) signalisierte, daß »der Krieg im All« nun möglich werde (ebd.). „Schon zu Ende dieses Jahrzehnts«, so hat es ein amerikanischer Rüstungsexperte prophezeit, »kann der Krieg im Weltraum Wirklichkeit sein« (ebd., S. 163).

Bereits die Mondfahrten hatten nach dem mißglückten Schweinebucht-Abenteuer gegen die kubanischen Kommunisten in den vom Rassenkampf und vom Vietnamkrieg beherrschten sechziger Jahren die Funktion gehabt, das Selbstgefühl der Amerikaner dadurch zu restituieren, daß man auf den »alten Pioniergeist« zurückgreifen und zu »new frontiers» vorstoßen konnte (Anders 1970, S. 160). Aber schon die Tatsache, daß die US-Astronauten auf dem Mond vergeblich darauf hoffen mußten, auf einer bis dato unentdeckten Südsee-Insel gelandet zu sein, auf der das Aufpflanzen des Sternenbanners Eingeborenen imponiert hätte (vgl. ebd., S. 76 f), verwies auf den Mangel dieser Frontier, an der es keine Feinde gab, gegen die man das Recht auf die eigene Landung hätte verteidigen können.

Mit der US-Raumfähre „Columbiax hat sich jedoch, so Reagan, seine neue Ära der Raumfahrt eröffnet (FR. 16.4.81, S. 1). Denn da nunmehr der Weltraum zur »neuen Arena für die Konfrontation der Supermächte« wird (Spiegel, 30.3.81, S. 156), gewinnt auch die Raumfahrt das dramatische Element, das den Kampf an dieser neuen Frontier erst interessant macht. Den Hauch des Abenteuerlichen erwirbt der Vorstoß ins All nämlich erst in 
dem Augenblick, wo sich der Amerikaner in den endlosen Weiten des Weltalls erneut als der einsame Cowboy der menschenleeren Prärien in Szene setzen kann, um im »Ein-MannRaumkreuzer« (ebd., S. 163) auf die Sowjets so Jagd zu machen wie einstmals auf die Indianer.

Die narzißtische Wut, die sie aufgrund der Verletzung ihres Selbstgefühls unter dem wachsenden Druck sich verschärfender wirtschaftlicher und politischer Konflikte ihres Landes entwickelten, verschoben die meisten Amerikaner auf die Russen, die Reagan auf der Grundlage seiner weltanschaulich begründeten und durch Pseudomythen verklärten Inszenierungen über ein Jahr lang zur Verkörperung des Bösen zu machen vermochte. Weil die Sowjets aber deshalb mit den gehaßten Selbstfragmenten identifiziert wurden, die die Amerikaner aus ihrer Wahrnehmung abgespalten haben, haben sie aufgrund ihrer »Furcht vor Vergeltung « 》erfolgungsängste« entwickelt (Klein 1962, S. 112), die in der Angst vor einem sowjetischen Überfall Gestalt annehmen und im »Krieg der Sterne« in so dramatischer Weise in Szene gesetzt wurden, in dem »für viele Hörer allzu überzeugend, die Invasion von Marsmenschen auf der Erde dargestellt» wurde (Stein 1981, S. 29).

Von dieser Vergeltungsangst suchen sich die Amerikaner, die Männern wie Reagan und Falwell Glauben schenken, dadurch zu befreien, daß sie in einem neuen Angriff auf die Welt die Vorzeichen dieser Weltkatastrophen-Phantasie umkehren, aufgrund dessen die Kommunisten als Ungeheuer von einem anderen Stern die Erde zu überfallen drohen: Wenn nämlich Reagan den beiden Astronauten der »Columbia« nach dem Erfolg ihres Testfluges versichert hat, daß die US-Bürger sich dank ihrer Leistung »wieder wie Giganten fühlen« können (Spiegel, 20.3.81, S. 236), dann inszeniert er die Amerikaner als die mächtigen Erdgeborenen, die die olympischen Götter angegriffen haben, indem »sie von ihren Bergspitzen Felsen und Feuerbrände in die Höhe« schleuderten (Ranke-Graves 1955, S. 115). Die sich derart als Giganten inszenierenden Amerikaner drohen in ihrer religiösen Sehnsucht nach dem Jüngsten Gericht einen alles Menschenleben zerstörenden Weltbrand zu entfachen, um sich durch die Vernichtung unseres Planeten selbst zu heilen. Denn gerade weil die Welt für diese Amerikaner kein eigenständiges Dasein mehr aufweist, vielmehr nur noch als »Rest der Welt« figuriert, als ein Selbst-Objekt, auf das sie ihre Aggression verschieben und das sie mit dem Bösen identifizieren, verspricht die Lösung dieses Wahnes nur noch der sich zur kosmischen Naturkatastrophe aufblähende Holocaust, für den Kirche und Staat die Massen zu mobilisieren schienen.

\section{Anmerkungen}

1 Wie es in Wirklichkeit um die Menschenrechte in El Salvador bestellt ist, zeigt der Völkermord jener von Reagan militärisch unterstützten Militärdiktatur, die die Herrschaft einer kleinen Oligarchie von Großgrundbesitzern mit Hilfe eines Terrors aufrechterhält, welcher von Polizei, Militär und den mit ihnen kooperierenden Todesschwadronen ausgeübt wird:

So wie die Terrortrupps »in Dörfer und Weiler« einfallen, um »auf brutalste Art Frauen, Alte, Kinder zu ermorden, die Häuser zu verbrennen, zu plündern und die Haustiere umzubringen (Spiegel, 9.3.81, S. 146), so findet man in der Hauptstadt San Salvador und Umgebung sjeden Morgen ... durchschnittlich ein Dutzend Gefolterte, Enthauptete, Vergewaltigte und Kastrierte, meistens mit unmißverständlichen Botschaften der Todesschwadronen versehen« (FR, 15.8.81, S. 3). Bei den unter der Zivilbevölkerung angerichteten Massakern, die »den Aufständischen den Rückhalt im Volk« entziehen sollen (Spiegel, 1.2.82, S. 102), reißt man den Opfern »Zunge und 
Ohren« aus, schneidet $\gg$ Schwangeren ... die Leibesfrucht« heraus, um sie »den Hunden zum Fraß vorzuwerfen (Spiegel 2.3.81, S. 131) oder wirft Säuglinge in die Luft und spießt sie mit dem Bajonett auf (vgl. Spiegel, 1.2.82, S. 100). Seit dem Oktober 1979 sind dieser Schreckensherrschaft über 30000 Menschen zum Opfer gefallen. Etwa 250000 Menschen befinden sich auf der Flucht vor diesem Terror (vgl. FR, 26.6.81, S. 13).

2 Daß Reagan sich mittlerweile zu Rüstungskontrollverhandlungen und auch zu einem Treffen mit Breschnew bereit erklärt hat, wirft die Frage auf, ob sich in der Gegenwart seine Konfrotationspolitik überhaupt noch gesamtgesellschaftlich durchsetzen läßt. Denn daß nicht nur Reagans Wirtschaftsprogramm nicht anschlägt, sondern auch seine Politik der Aufrüstung durch den wachsenden politischen Protest in Frage gestellt wird, der sich aufgrund der im Frïhjahr dieses Jahres in den USA entstandenen Friedensbewegung entwickelt hat, verweist auf das den autoritätssgebundenen Charakterdispositionen entgegengesetzte genuin liberale Widerstandspotential der Amerikaner, eine Tatsache, die jedoch nichts an dem erklärungsbedürftigen Sachverhalt ändert, daß die irrationale Konfrontationspolitik Reagans über ein Jahr lang eine Massenbasis hatte.

3 Wenn im Folgenden das Problem der Kirche in Amerika erörtert wird, so geht es dabei um das Problem des Zerfalls der Religion, die zu einer Weltanschauung verkommt, die, wrational und irrational zugleich« (Lorenzer 1981a, S. 119), an »eine sozial bedingte Persönlichkeitsirritation anknüpft (ebd., S. 121), um den Einzelnen unter Ausnutzung seiner infantilen Fixierungen in das »organisierte Bewußtsein einer Masse« einzubetten und ihn damit »von seiner asozialen Isolierung« zu erlösen (ebd., S. 122). Evangelisation wird derart als »weltanschauliche Massenbildung» wirksam (ebd., S. 127), wenn die Fersehprediger der fundamentalistischen Kirche ihre Gläubigen für Reagans Politik zu mobilisieren sich bemühen - oder aber der Papst sich auf seinen Auslandsreisen als massenwirksamer Volksführer in Szene setzt, den die Massenmedien als Super-Star feiern, auch wenn er nach wie vor die Ehescheidung verbietet und jede Form künstlicher Geburtenregelung verdammt (vgl. König 1981).

Anders als im Falle dieser zur Weltanschauung verflachten Religion, die an die Persönlichkeitsdefekte des Einzelnen anknüpft, um jede »soziale Unruhe« sogleich »durch eine irrationale Lösung« stillzustellen (Lorenzer 1981a, S. 128), bringen die amerikanischen Christen, die sich wie die Katholiken mit ihren in El Salvador verfolgten Glaubensbrüdern solidarisieren (vgl. Spiegel, 19.4.82, S. 167) und für das »Einfrieren der Atomrüstung « demonstrieren (ebd, , S. 160), die individuellen Bedürfnisse und Sorgen der durch die Politik Reagans sich betroffen fühlenden Menschen zum Ausdruck, die nicht länger bereit sind, das Problem der Waffenkontrolle »den Haigs und Weinbergers« zu überlassen (ebd., S. 168). In diesem Fall ist das Ergebnis nicht Massenbildung, sondern eine »Kollektivbildung (Lorenzer 1981a, S. 131), die es ermöglicht, die »Irritationen des Alltagslebens« (ebd.) im Dienste einer gesellschaftsverändernden politischen Praxis nutzbar zu machen.

4 Der Begriff des »Fußbodens« ist nicht einfach Karikatur, sondern verleiht einer zur grauenhaften Karikatur getonnen Wirklichkeit Ausdruck. Denn gerade die Männer, die im Alltagsleben so oft den »Reinigungszwang neurotischer Hausfrauen« (Abraham 1924, S. 124) belächeln und verspotten, funktionalisieten die dem weiblichen Geschlecht zugeschriebene »Herrschsucht« (ebd.), die sich mit Hilfe von Putzeimer und Staubsauger behauptet, im eigenen Interesse, um mit ihter Hilfe die namenlose Gewalt zu verleugnen, die sie selbst auf gesellschaftlicher und politischer Ebene in Szene setzen, indem sie die von den Menschen bewohten Städte und Landschaften zu einem vom Ungeziefer des Lebendigen bewohnten Fußboden machen, den sie mit ihten »Bombenteppichen zudecken.

5 Zur Unterscheidung des Über-Ichs als einer einschränkend, verbietenden Instanz und des IchIdeals als einer Instanz der Wunscherfüllung vgl. Lampl-de-Groot 1963.

6 Die Tatsache, daß »unsre Erfahrungen mit dem Nationalsozialismus ... uns allzusehr darauf fixiert« haben, »die Gefahr der Demagogen in ihren ideologischen Konzepten und ihrer politischen Rhetorik zu suchen, obgleich der ... Faschismus uns schon auf die Verführungsmacht der Bilder hätte hinweisen können« (Lorenzer 1981b, S. 16), macht uns darauf aufmerksam, daß das Massen- 
individuum, sin der nachinfantilen Vergesellschaftung« nicht nur über die ihm angebotene »Weltanschauung (Lorenzer 1981a, S. 121), sondern auch über die ihm präsentierte Bildervelt sozialisiert wird, die die Herrschenden zur Durchsetzung ihrer Interessen inszenieren: $\gg$ Man erinnere sich des gewaltigen sinnlichen Repertoires der nationalsozialistischen Inszenierungen, der ausgesonnenen Rituale mit der Blutfahne, der starren Schematik der Aufmärsche ... der systematischen Tilgung konkurrierender Bilder und Bildproduktionen durch die vom Propagandaministerium gleichgeschalteten 'Kultur'-Instanzen« (ebd,. S. 168).

7 So wie schon auf dem Weg in die Sklaverei nicht weniger als 10 Millionen Schwarze bei ihrem Transport nach Nordamerika umkamen (vgl. Jacobs, Landau, Pell 1971, S. 69), so wurden die schätzungsweise 10 Millionen Indianer, die noch im frühen 17. Jahrhundert den nordamerikanischen Kontinent besiedelten, bis auf den heutigen Tag auf 850000 dezimiert (vgl. Spiegel, 19.11.79, S. 212).

8 Auf den Sachverhalt, daß die »'formgebenden' Körpervorgänge der Mutter ... von keiner anderen Art als die formgebenden Handgriffe des Arbeiters sind, weil die »Auseinandersetzung des Arbeiters mit der 'äußeren Natur' ... in einem praktischen Umgang« realisiert wird, »der dem praktischen 'formgebenden' Umgang der Mutter in der Mutter-Kind-Dyade in vollem Umfang vergleichbar ist«, hat Lorenzer in der von ihm entworfenen materialistischen Sozialisationstheorie aufmerksam gemacht (Lorenzer, 1972, S. 50 f).

\section{Literaturverzeichnis}

Abraham 1924: Karl Abraham, Versuch einer Entwicklungsgescbichte der Libido auf Grund der Psychoanalyse seelischer Störungen (1924). In: Karl Abraham (1969), Psychoanalytische Studien, Bd. I, Frankfurt 1971, S. 113-183

Adorno u.a. 1953: Theodor W. Adorno u.a., Der autoritäre Cbarakter. Studien über Autorität und Vorurteil (1953), Amsterdam 1977

Adorno 1973: Theodor W. Adorno, Studien zum autonitären Charakter Frankfurt 1973

Alsheimer 1966: Georg W. Alsheimer, Amerikaner in Vietnam, Das Argument, Februar 1966, S. 243

Anders 1970: Günther Anders, Der Blick vom Mond, München 1970

Biegert 1976: Claus Biegert, Seit 200 Jabren obne Verfassung. USA: Indianer im Widerstand(1976), Reinbek 1979

Erikson 1950: Erik H. Erison, Kindheit und Gesellschaft (1950), Stuttgart 1971

Ferguson, Rogers 1981: Thomas Ferguson, Joel Rogers, Der Sieg Reagans, Prokla Heft 44, 1981, S. $57-$ 86

Fiedler 1968: Leslie A. Fiedler, Die Rückkehr des verschwundenen Amerikaners (1968), Frankfurt 1970

Freud 1911: Sigmund Freud, Psycboanalytische Bemerkungen uber einen autobiograpbisch beschriebenen Fall von Paranoia (1911), S. Freud Studienausgabe Bd. VII, Frankfurt, S. 133-203

Fromm 1970: Erich Fromm, Analytische Sozialpsycbologie und Gesellschaftstheorie (1970), Frankfurt 1972

Horkheimer, Adorno 1944: Max Horkheimer, Theodor W. Adorno, Dialektik der Aufklärung (1944), Frankfurt 1971

Jacobs, Landau, Pell 1971: Paul Jacobs, Saul Landau, Eve Pell, Brïder, sollen wir uns unterwerfen? Die verleugnete Geschichte Amerikas (1971), München 1975

Kilian 1979: Martin Kilian, Die Genesis des Amerikanismus. Zum Verbältnis von amerikanischer Ideologie und amerikanischer Praxis 1630-1789, Frankfurt 1979

Klein 1962: Melanie Klein, Das Seelenleben des Kleinkindes und andere Beiträge zur Psychoanalyse (1962), Reinbek 1972

Kohut 1975: Heinz Kohut, Die Zukunft der Psychoanalyse, Frankfurt 1975 
König 1981: Hans-Dieter König, Rituale der welttichen und der geistlichen Gewalt. Zu den öffentlichen Gelöbnissen und zum Papstbesuch 1980, Psyche 1981, S. 642-656

Lampl-de-Groot 1963: Jeanne Lampl-de-Groot, Ich-Ideal und Über-Ich, Psyche 1963, S. 321-332

Lorenzer 1972: Alfred Lorenzer, Zur Begründung einer materialistischen Sozialisationstheorie, Frankfurt 1972

Lorenzer 1981a: Alfred Lorenzer, Das Konzil der Buchbalter. Die Zerstörung der Sinnlichkeit. Eine Religionskritik, Frankfurt 1981

Lorenzer 1981b: Alfred Lorenzer, Literatur als politischer Eingriff, Manuskript

Marcuse 1968: Herbert Marcuse, Aggressivität in der gegenwärtigen Industriegesellschaft. In: Aggression und Anpassung in der Industriegesellschaft, Frankfurt 1968, S. 7-29

Mitscherlich und Mitscherlich 1967: Alexander Mitscherlich und Margarete Mitscherlich, Die Unfäbigkeit zu trauem. Grundlagen kollektiven Verbaltens, München 1967

Ranke-Graves 1955: Robert von Ranke-Graves, Griechische Mythologie. Quellen und Deutung, Bd. 1. (1955), Reinbek 1976

Reagan 1979: Ronald W. Reagan, Unforgettable Jobn Wayne, Reader's Digest, October 1979, S. 114 119

Reagan 1980: Ronald W. Reagan, Die gesammelten Worte des R.W. Reagan (1980), München 1981 Reagan 1981: Ronald W. Reagan, Ein Neubeginn für Amerika. Ansprache des neuen Präsidenten Ronald Reagan zu seinem Amtsantritt am 20.1.1981. International Communication Agency. Embassy of the United States of America, Bonn, S. 7-12

Segal 1964: Hanna Segal, Melanie Klein. Eine Einfübrung in ibr Werk (1964), München 1974

Seeßlen, Weil 1979: Georg Seeßlen, Claudius Weil, Western-Kino. Gescbicbte und Mythologie des Western-Films. Bd. 1, Reinbek 1979

Stein 1981: Howard F. Stein, USA: Krieg aus Selbstmitleid? Psychologie heute, Februar 1981, S. 2229 